\title{
Adaptation of transferrin protein and glycan synthesis
}

\author{
G. de Jong ${ }^{\mathrm{a}, \mathrm{b}}$, W.L. van Noort ${ }^{\mathrm{a}}$, R.A. Feelders ${ }^{\mathrm{a}}$, C.M.H. de \\ Jeu-Jaspars $^{\mathrm{a}}$ and H.G. van Eijk ${ }^{\mathrm{a}}$
}

${ }^{a}$ Department of Chemical Pathology, Erasmus University, Rotterdam and ${ }^{b}$ Department of Internal Medicine II. University Hospital Dijkzigt Rotterdam, Erasmus University, Rotterdam (The Netherlands)

(Received 24 December 1991; revision received 14 August 1992; accepted 9 September 1992)

Key words: Transferrin; Glycosylation; Glycan; Pregnancy; Oral contraceptives; Iron deficiency; Rheumatoid arthritis; Microheterogeneity

\section{Summary}

We report the patterns of variability in transferrin structure in pregnancy, iron deficiency anemia, women using oral contraceptives, nonanaemic rheumatoid arthritis, iron deficient rheumatoid arthritis and anemia of the chronic diseases. Changes in microheterogeneity were assessed by crossed immuno isoelectric focusing of serum transferrin. Intra-individual variation in the control group was minimal. Equally, inter-individual variation in controls and groups with established stable disease was very limited. In pregnancy an increase in transferrin concentration was accompanied by redirection of glycan synthesis to the highly sialylated and highly branched glycans, an effect also shown in women using oral contraceptives. Iron deficiency anemia was accompanied by increased protein core synthesis without the large shifts in the microheterogeneity pattern as seen in pregnancy at similar transferrin concentration. In contrast to this, rheumatoid arthritis was accompanied by decreased protein synthesis while the microheterogeneity pattern shifted significantly towards the highly branched glycans. Interpreted in the respective pathophysiological contexts results show that: (1) N-linked glycosylation of transferrin is a strictly controlled process, both in the physiological states and in disease. (2) Microheterogeneity is determined independently from transferrin protein synthetic rate. (3) Provisionally

Correspondence to: G. de Jong, Department of Chemical Pathology, Erasmus University Rotterdam, P.O. Box 1738, 3000 DR Rotterdam, The Netherlands.

Abbreviations: CIEF, Crossed immuno-isoelectric focusing; Tf, transferrin; RA, rheumatoid arthritis; $A C D$, anemia of the chronic disorders. 
observed changes in the glycosylation can modulate the biological activity of the glycoprotein and as a result redirect internal iron fluxes. This proposition can be applied to altered iron metabolism in both pregnancy, oral contraceptives and rheumatoid arthritis. Changes are not operative in iron deficiency because qualitatively iron metabolism is not altered in this state.

\section{Introduction}

Transferrin ( $\mathrm{T} f$ ) plays a key role in iron homeostasis in vertebrates, and possibly also in some invertebrates, as an iron transporting protein. In humans structural determinants of the protein have been extensively investigated (for reviews see Refs. 1-3). Tf is a bilobed monomeric protein, the lobes corresponding to the chemically defined homologous $\mathrm{N}$-terminal and $\mathrm{C}$-terminal domains. The C-terminal domain carries the two $\mathrm{N}$-linked complex type oligosaccharide chains, which in principle can be any one of a wide array of structures differing in degree of branching and terminal carbohydrate composition [3]. Tf is a convenient model for studying $\mathrm{N}$-glycosylation because it is present in relatively high concentrations in many body fluids, its number of glycosylation sites is known and possible glycan structures have been structurally defined. Crossed immuno isoelectric focusing of iron saturated serum samples is a sensitive method of assessing this variability [4], providing an accurate account of the proportions of the fractions characterized by the number of sialic acids. Variation in glycan structure due to changes in neutral carbohydrate residues is not directly measured, but indirectly most of this variability is registered since a good estimation of the relative amounts of the Tfs that can be distinguished on the basis of the number of branches on the two glycans attached to the Tf molecule is provided by this technique. Remaining variation due to electrophoretically neutral variation in glycan structure hidden from this technique is accepted, albeit anticipated to be minimal in serum samples in view of uniformity in glycan core structure and the existence of potent scavenging systems for asialoglycoproteins $[3,4]$. Carbohydrate analysis of isolated fractions confirming the basis for this separation has been published recently [5].

Even in cases related to an inborn error of metabolism directly involving $\mathrm{N}$ glycosylation [6,7], attempts to electrophoretically identify unique subfractions in patients sera pathognomonic for the existence of a particular disease, have been unsuccessful [8-10]. As opposed to this, our approach has been to describe and compare whole spectra of microheterogeneous forms of a glycoprotein with respect to both percentual representation and to actual concentrations of fractions in serum. This paper reports on the patterns of variability in Tf structure in several conditions (Table I) and in doing so aimed to identify factors that contribute to the regulation of Tf glycosylation. As explained in the discussion, through the respective denominations of (patho-) physiological circumstances this wide range of groups was expected to shed light on regulatory aspects of Tf protein and glycan synthesis and along the same line we expected to obtain some clues on the role of Tf microheterogeneity in iron metabolism, a field which has as yet received little attention. 
TABLE I

Investigated groups

\begin{tabular}{llr}
\hline Designation & Group description & $n$ \\
\hline C & Control males & 8 \\
N & Control females & 13 \\
I & Pregnant women, week 8-16 & 12 \\
II & Pregnant women, week 17-26 & 10 \\
III & Pregnant women, week 27-40 & 18 \\
AT & Pregnant women, a terme week 36-40 & 9 \\
PP & 3 days post partum & 9 \\
PPM & 5 weeks postpartum & 7 \\
O & & 7 \\
Fe & Women on oral contraceptives & 9 \\
RA-NA & Iron deficiency anemia & 6 \\
RA-Fe & Rheumatoid arthritis, not anemic & 6 \\
RA-ACD & Rheumatoid arthritis, iron deficiency & 6 \\
\hline
\end{tabular}

\section{Materials and Methods}

\section{Patients}

After acquiring informed consent, blood was obtained from 21 healthy volunteers, 8 male and 13 female (all women in first half of menstrual cycle), 56 pregnant women attending the University Hospital Obstetrical Clinic, 7 women using oral contraceptives, 9 women with iron deficiency anemia and 18 female patients with rheumatoid arthritis (RA) fitting the revised ARA-criteria [11]. Anemic pregnant women and pregnant women with a latent iron deficiency were excluded by hemoglobin and serum ferritin measurements. The course of pregnancy and delivery was uncomplicated in all cases. All 18 female RA patients had active disease and used nonsteroidal anti-inflammatory drugs, as well as long-acting anti-rheumatic drugs. Other causes of anemia were excluded by history taking (present or past ulcer disease or other complaints or signs of gastrointestinal diseases, hypermenorrhoea and haematuria), negative stools for occult blood, a normal vitamin B12, folic acid, Coombs test, creatinine clearance and absence of microscopic hematuria. The female RA patients were divided into three groups of six: nonanemic patients, patients with iron deficiency anemia and patients with anemia of the chronic diseases. This distinction has been made on established criteria based on hemoglobin, serum $\mathrm{Tf}$ and serum ferritin measurements $[12,13]$.

\section{Sample treatment}

Venous blood samples were obtained between 10:00 and 12:00 h in glass tubes not containing anticoagulant. Omitting the anticoagulant is a relevant part of the pro- 
cedure as we and others have found that the addition may influence the isoelectric focusing [14]. After clot retraction, the sample was centrifuged for $20 \mathrm{~min}$ at $1,200 \times g$ and $4^{\circ} \mathrm{C}$. The serum was used immediately or stored at $-20^{\circ} \mathrm{C}$ or $-70^{\circ} \mathrm{C}$ until use. Before storage or application on the gels complete iron saturation of the Tf was ensured by mixing $100 \mu \mathrm{l}$ serum with $5 \mu \mathrm{l}$ of $0.5 \mathrm{M} \mathrm{NaHCO}_{3}$ and $3 \mu \mathrm{l}$ of 10 $\mu \mathrm{M} \mathrm{Fe}(\mathrm{III})$ citrate which was then left at room temperature for $1 \mathrm{~h}$. Rabbit anti human Tf-anti sera were home made and have been compared favourably with anti sera from Dako, Denmark.

\section{Crossed immuno-isoelectric focusing (CIEF)}

This method involves two phases combining two electrophoretic techniques; the first establishing separation of proteins of interest, the second run perpendicularly to the first for identification and quantification purposes.

In the first phase the Tf subfractions were separated on polyacrylamide gelstrips on an immobilized $\mathrm{pH}$-gradient by isoelectric focusing. Strips, $4 \mathrm{~mm}$ wide, cut from an Immobiline Dry Plate, pH 4.9-6.1 (purchased from LKB-Produkter AB, Bromma, Sweden), which had previously been rehydrated in aqua bidest to a final weight of $20 \mathrm{~g} / \mathrm{gel}$, were placed on a thermostatic template kept at $10^{\circ} \mathrm{C}$. Anode and cathode strips soaked in $10 \mathrm{mM}$ glutamic acid and $10 \mathrm{mM} \mathrm{NaOH}$, respectively, were placed over the ends of the immobiline strips. Two milliliters of the iron-saturated serum was applied at the cathodal side of the strips. A voltage of $1 \mathrm{kV}$ was applied during $18 \mathrm{~h}$ at $10^{\circ} \mathrm{C}$.

For the second phase of the electrophoretic procedure, $60 \mathrm{ml}$ of a $1 \%(\mathrm{w} / \mathrm{v})$ solution of Agarose-M (LKB) in 24-mM sodium barbiturate-HCl buffer, $\mathrm{pH} 8.7$, prepared at $100^{\circ} \mathrm{C}$ were cooled to $55^{\circ} \mathrm{C}$, whereupon a preheated $\left(55^{\circ} \mathrm{C}\right)$ amount of the rabbit anti-human Tf preparation was added and thoroughly mixed with the agarose solution. This mixture was poured onto a preheated glass plate $(130 \times 260$ $\mathrm{mm}$ ) placed on top of a second glass plate kept at $50^{\circ} \mathrm{C}$. Then the Immobiline strips from the isoelectric focusing step, having been soaked for $15 \mathrm{~min}$ in the electrophoresis buffer, were placed upside down on the agarose surface. The crossed immunoelectrophoresis is carried out in the 24-mM barbiturate- $\mathrm{HCl}$ buffer during $4 \mathrm{~h}$ at $10^{\circ} \mathrm{C}$ at a voltage of $12-13 \mathrm{~V} / \mathrm{cm}$. All determinations were done in duplicate. The fractions were successively quantitated via measurement of the areas enclosed by the immunoelectrophoretic peaks. In Fig. 1 a representative example is shown of a microheterogeneity pattern.

Tf concentrations were measured turbidimetrically [15] using the Boehringer ES22. For this Tf was immunoprecipitated with sheep-anti-human-Tf (purchased from Boehringer) in an $0.1 \mathrm{M} \mathrm{Na} \mathrm{HPO}_{4} / \mathrm{KH}_{2} \mathrm{PO}_{4}$ buffer, $\mathrm{pH} 7.5$, containing $4 \%$ polyethylene glycol. Ferritin concentrations were measured by ELISA.

Known $\mathrm{Tf}$ functions are concentration dependent processes. Therefore concentrations rather than ratios of subfractions are probably more important in the biological functions of Tf. Thus in all groups individual concentrations have been equated from fractional ratios and total Tf concentration. In pregnancy and other groups this adjusts the effects to its physiological proportions, as fraction ratios (assuming unaltered, similar serum half lives) justly describe the adaptation of syn- 


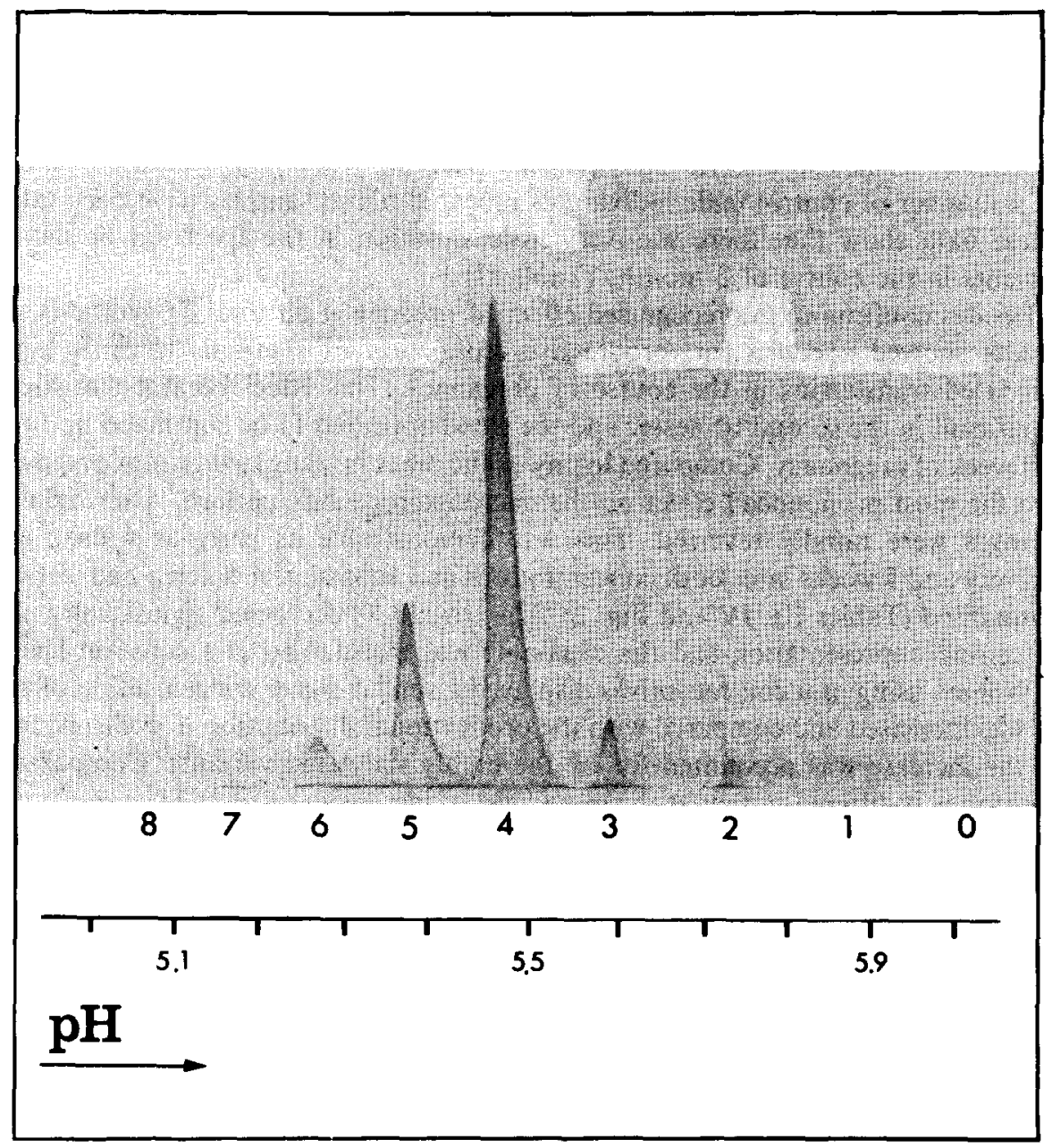

Fig. 1. CIEF serum sample. Numerals indicate number of sialic acid residues per protein molecule. Cathode at right and bottom for first and second phase, respectively.

thesis, but in conditions where total protein synthesis increases as well, this presentation may overstate the possible physiological implications.

\section{Statistical analysis}

Total Tf concentrations were compared using the two tailed Student's $t$-test and non-parametric data by the Mann-Whitney U-test. Coefficients of correlation were compared using Spearman's test. 


\section{Results}

In Tables II-IV a full account of the results is given. Groups of patients have been designated as explained in Table I. Between males and control females no significant differences existed in diferric $\mathrm{Tf}$ spectrum, total and fractional $\mathrm{Tf}$ concentrations. Of a number of control male individuals repeated blood samples have been taken. These data show that there was very little variation in the spectrum of sialo-Tf variants in the course of 3 months (Table II).

Besides confirming the recognized effect of pregnancy on total Tf synthesis, our results showed a consistent, progressive redirection of synthesis towards highly sialylated transferrins in the course of pregnancy. This effect became statistically significant in the second trimester and the trend appeared to be continued up to the last week of pregnancy. Compared to any of the other investigated groups pregnancy had the most pronounced effect on the microheterogeneity pattern. After delivery, changes were rapidly reversed. This was demonstrable as early as 4 days after delivery and 5 weeks later both concentrations and subfraction-pattern had virtually normalised (Tables III, IV and Fig. 2). The trisialo-Tf decreased significantly in its percentual representation and the disialo-Tf was maintained at a constant level.

Women using oral contraceptives displayed a similar trend: concentration of total Tf was increased and compared with the proportional distribution in controls, more of the increase was accounted for by the highly sialylated variants. Compared to pregnancy it differed in that at the same level of increment of total Tf concentration the change in the proportional representation was less marked than in pregnancy. Iron deficiency was accompanied by an increased total $\mathrm{Tf}$ concentration, the group average being risen by $48.2 \%$. Relative proportions were shifted considerably less towards the highly sialylated fractions, reaching statistical significance only for the

\section{TABLE II}

Variation of pattern in control male individuals

\begin{tabular}{lllllllll}
\hline & \multicolumn{7}{c}{ Proportional distribution of sialo-Tfs (\%) } \\
\cline { 2 - 8 } & mono & di & tri & tetra & penta & hexa & hepta \\
\hline $\begin{array}{l}\text { Average } \\
\quad(n=4)\end{array}$ & 0.0 & 2.6 & 7.4 & 66.1 & 18.9 & 4.9 & 0.1 \\
$\begin{array}{l}\text { Maximum } \\
\text { inter-individual } \\
\text { variation } \\
\quad(n=54)\end{array}$ & 0.0 & 1.1 & 2.1 & 2.6 & 1.5 & 1.9 & 0.1 \\
$\begin{array}{l}\text { Maximum } \\
\text { intra-individual } \\
\text { variation } \\
(n=12)\end{array}$ & 0.0 & 0.2 & 0.5 & 0.8 & 0.6 & 0.3 & 0.1 \\
\hline
\end{tabular}

Proportional distribution of sialo-Tf fractions in four male controls in the course of 3 months. Results expressed as percentages of total amount. Total variation of the spectrum within a single individual ranged from 1.1 to $2.3 \%$. 


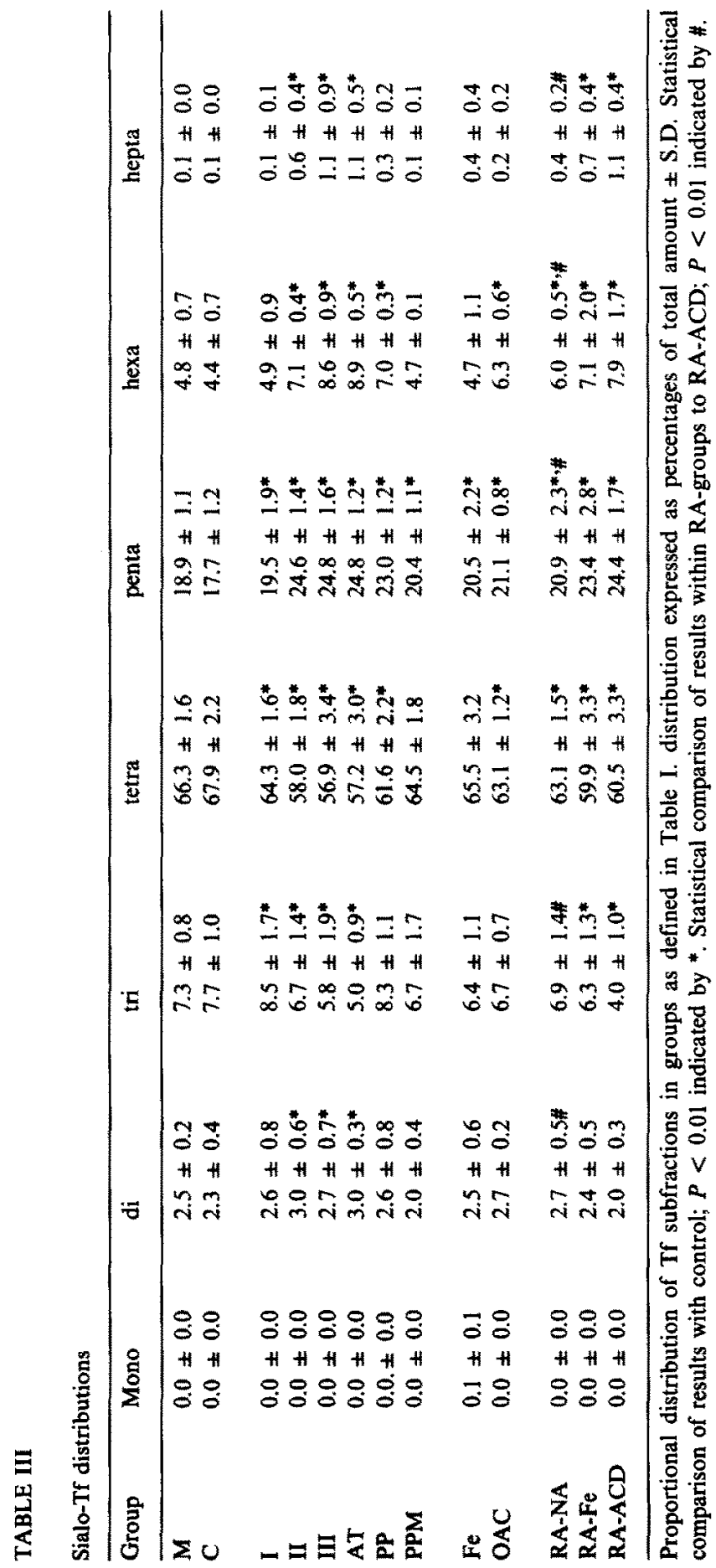




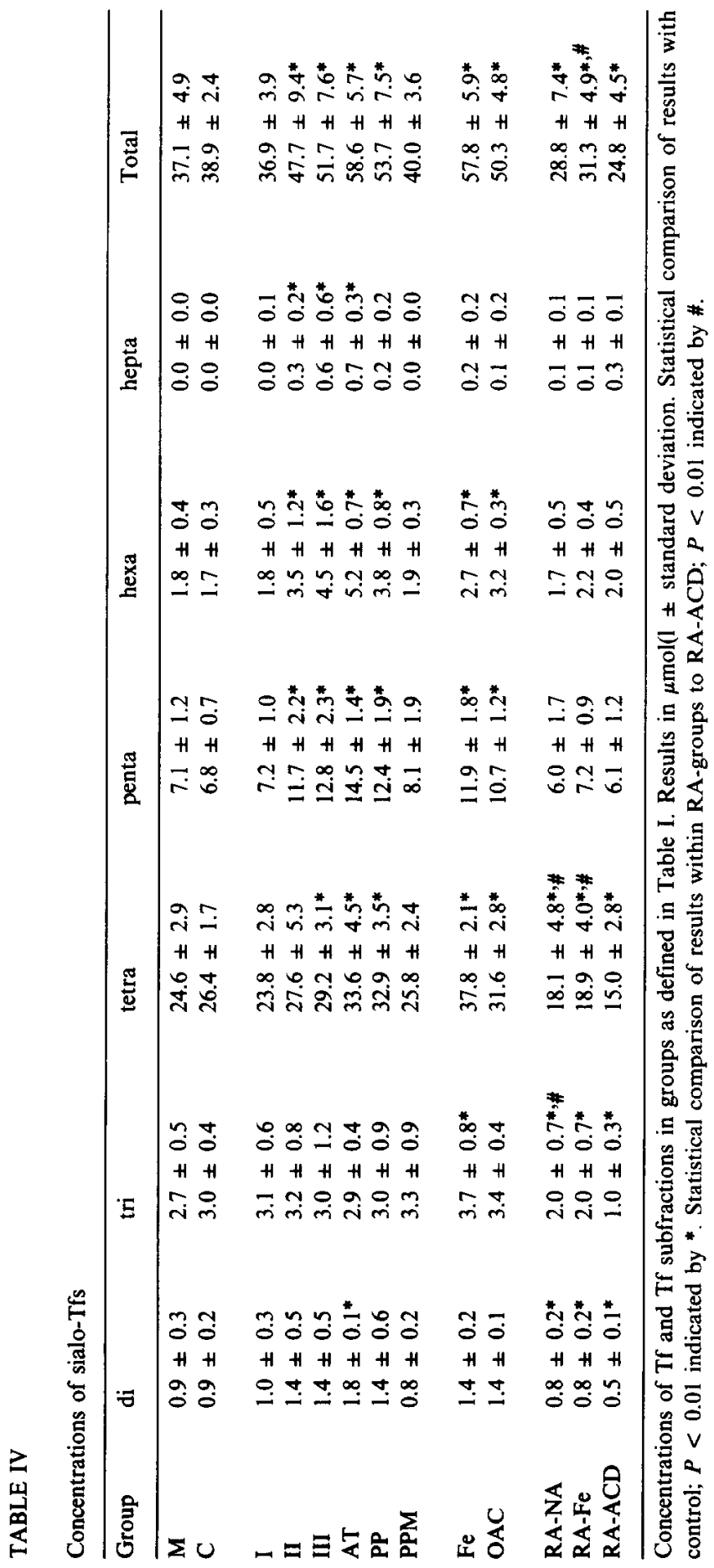


(2)

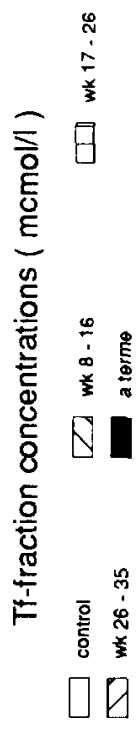<smiles>[SiH3]</smiles>

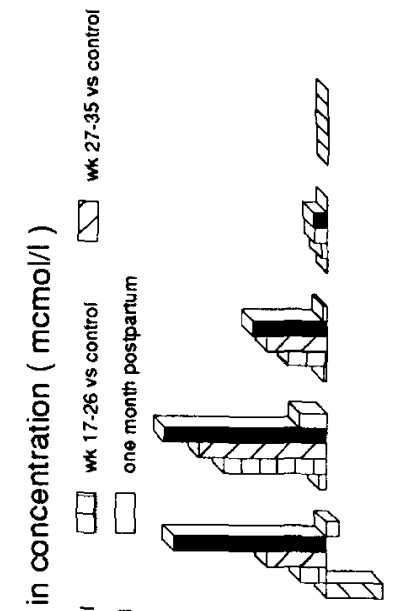

g d
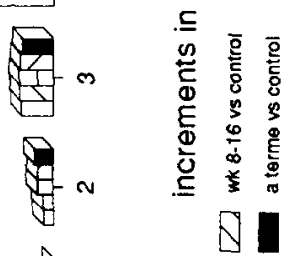

8 م

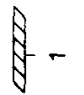

8<smiles>[CH]=C</smiles>
$\stackrel{1}{b}$ is i

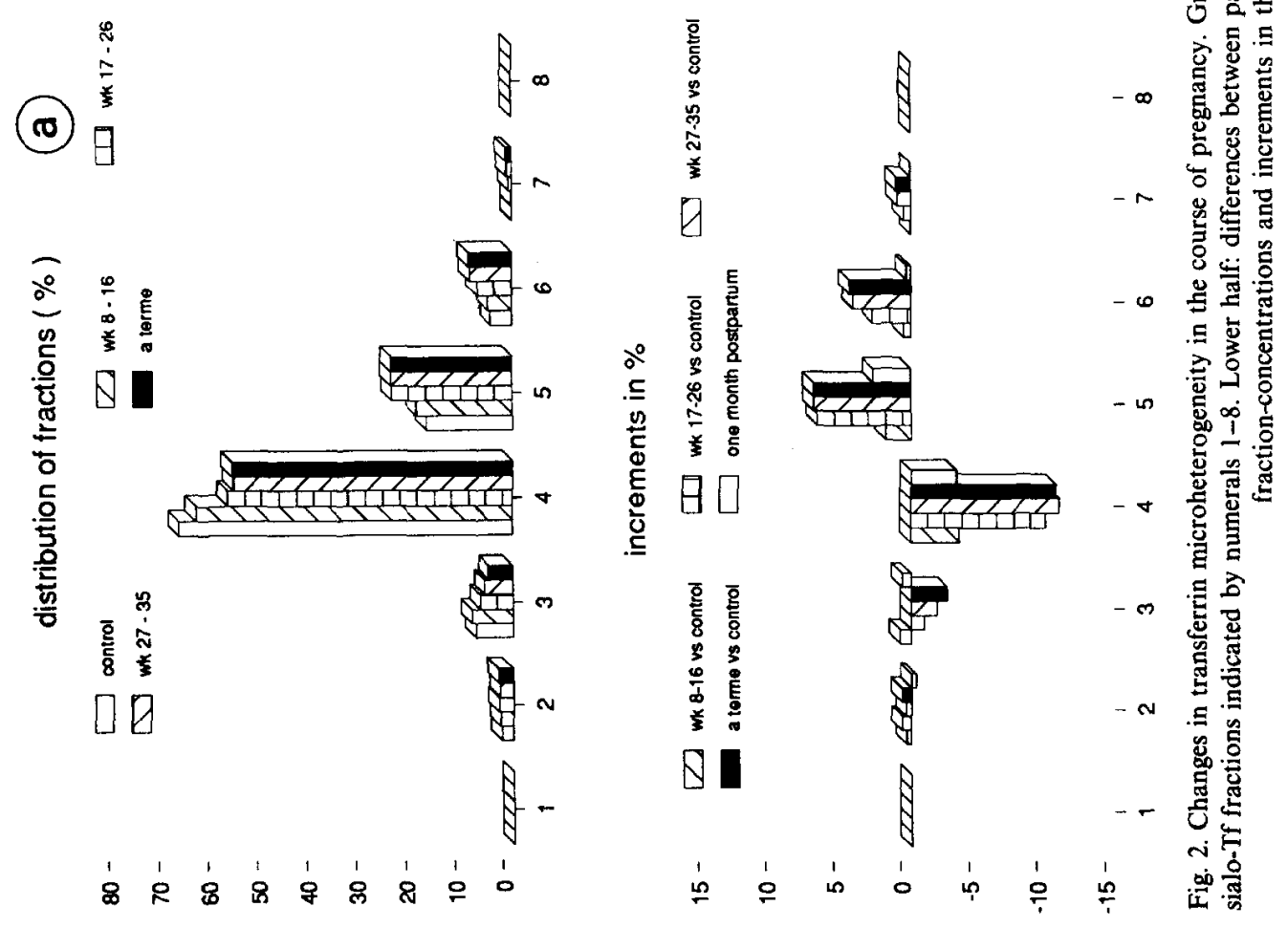


pentasialo-Tf. Compared to pregnancy total Tf concentration in iron deficiency was higher and most of the actual increment in concentration was due to increased production of tetrasialo-Tf.

The patients with active RA generally had a lowered total $\mathrm{Tf}$ concentration. The decrease was less in the subgroup with concomitant iron deficiency, but still significantly low compared to healthy controls. The microheterogeneity pattern was again changed in favour of the highly sialylated $T f$ s. This change was least pronounced in the nonanemic RA patients and most pronounced in the anemia of the chronic disorder (ACD) group, with the iron deficiency group occupying the intermediate position. The synthetic balance was shifted to the extent that in both the iron deficiency and ACD-subgroups the concentrations of penta, hexa and heptasialoTfs were not significantly depressed compared to controls, while the total Tf concentration in these groups was diminished by $15.1 \%$ and $38.8 \%$, respectively. The tetrasialo-Tf was significantly depressed in both proportional representation and in concentration in all RA subgroups; and a similar effect is observed for the di- and trisialo-Tf.

\section{Discussion}

A global shift of synthesis towards highly sialylated and more branched chains in all conditions understates the information available from these results. Reviewing results in the context of contemporary understanding of both the underlying pathophysiological conditions and the mechanism from which microheterogeneity evolves disclosed additional conclusions.

\section{Identification of factors influencing microheterogeneity patterns}

Results from pregnant women (Tables III, IV and Fig. 2) clearly show adaptation of Tf glycan synthesis in the course of pregnancy. The tetrasialo-Tf concentration increases despite its proportional decrease, the major part of the increase in the total Tf concentration during pregnancy, however, is due to the increase in the concentration of highly sialylated Tfs. No significant change in the concentration of the desialylated Tfs is registered, which is consistent with an adequately functioning asialoglycoprotein scavenger system.

Endocrinological influences on the microheterogeneity pattern, were further ascertained comparing women on oral contraceptives and women suffering from an isolated iron-deficiency anemia with controls and pregnant women (Fig. 3). In women on oral contraceptives the pattern changes in the same direction as in pregnancy, as such confirming the notion that oral contraceptives can influence the glycosylation system with reference to Tf [16]. Compared to pregnancy, at the same level of increment of total $\mathrm{Tf}$ concentration, the change in proportional representation was less marked in women using oral contraceptives. This difference in pattern may be related to the difference in the estrogen to progesterone ratio as the latter is higher in pregnant women than in the OAC group. Progesterone has been reported to influence glycosylation, data on estrogen are limited to stimulation of total Tf synthesis. Therefore the above described difference could be explained on the basis of 


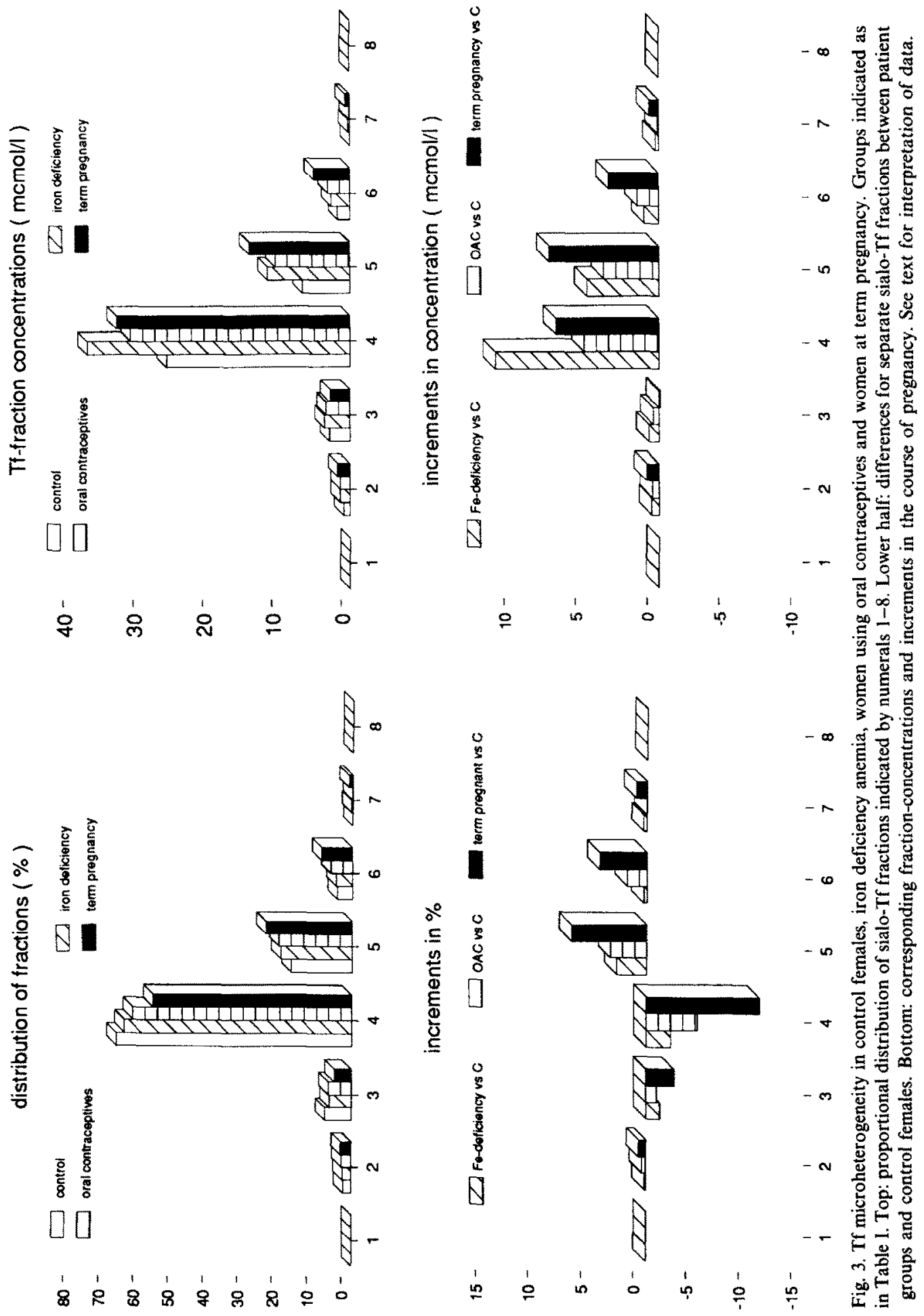


these assumptions. Alternative explanations lie in the preponderance of other hormones in pregnancy. Prolactin can probably be excluded in view of the rapid normalization of the microheterogeneity pattern after delivery in lactating women, but as such endocrinological influences are certainly significant.

As reflected in clinical chemical parameters, the rheumatoid arthritis groups comprise a spectrum of disease activity [13,17]; in order of increasing disease activity the nonanaemic, the iron deficient and the ACD group. Changes in microheterogeneity are again similar in direction, but this time protein synthesis is significantly reduced as a concomitant feature of the acute phase reaction. As the alteration in glycosylation was more pronounced in the anemic groups with the ACD group displaying the greatest change in pattern, change in glycosylation of $\mathrm{Tf}$ in RA appears to be correlated with disease activity (Fig. 4), confirming conclusions drawn from studies on concanavalin A binding patterns of $\alpha_{1}$ acid glycoprotein [18]. The decreased concentration of the disialo- and trisialo-Tf in RA is significant and this also appears to correlate with disease activity. The latter can be interpreted as resulting from the activation of the asialoglycoprotein scavenging system in the acute phase reaction, thus working at higher efficiency. The higher avidity of this system as such for desialylated tri- and tetra-antennary glycans [19] may be an additional factor.

\section{Regulatory aspects of $\mathrm{N}$-glycosylation and protein synthesis}

Reviewing glycan synthesis being beyond the scope of this article [20-23] it will suffice here to briefly indicate the context for reference. Evolutionarily glycan synthesis is one step up the ladder compared to protein synthesis, particularly in its coding mechanism. Through both the possibility of branching and a combination of anomerous configurations and types of bonding between identical sets of components, a vast combination of structural determinants is created, as in other macromolecular synthetic machineries, but glycans are distinct from polypeptides and nucleic acids in the comparatively small size at which analogous astronomic diversity is attained. It is also distinct from nucleic acid and polypeptide synthesis in that it is not coded for by an accurate template mechanism. In essence glycan structure is coded for by intrinsic information laid down in the combination of primary, secondary and tertiary characteristics of the polypeptide core structure, in conjunction with characteristics of enzymes involved in the synthetic machinery as well as the interaction with the matrix in which it is processed. Consequently outcome is not explicitly defined, at the cost of loss of uniformity, hence the phenomenon of microheterogeneity.

From this understanding it evolved that amongst others the results of the CIEF of serum Tf can be appreciated as a means of assessment of the accuracy of control over the glycosylation system. Our results are based on a technique that provides a very accurate and reproducible measure of $\mathrm{Tf}$ microheterogeneity. In vicw of the complexity of the synthetic system and its control, it is clear how little variation there in fact is in the oligosaccharide-structures attached to this particular protein. Although some heterogeneity is still hidden from isoelectric focusing, only 9 different fractions can be distinguished in iron saturated human serum. As shown in Table II, in single individuals the variation in this microheterogeneity is minimal. 


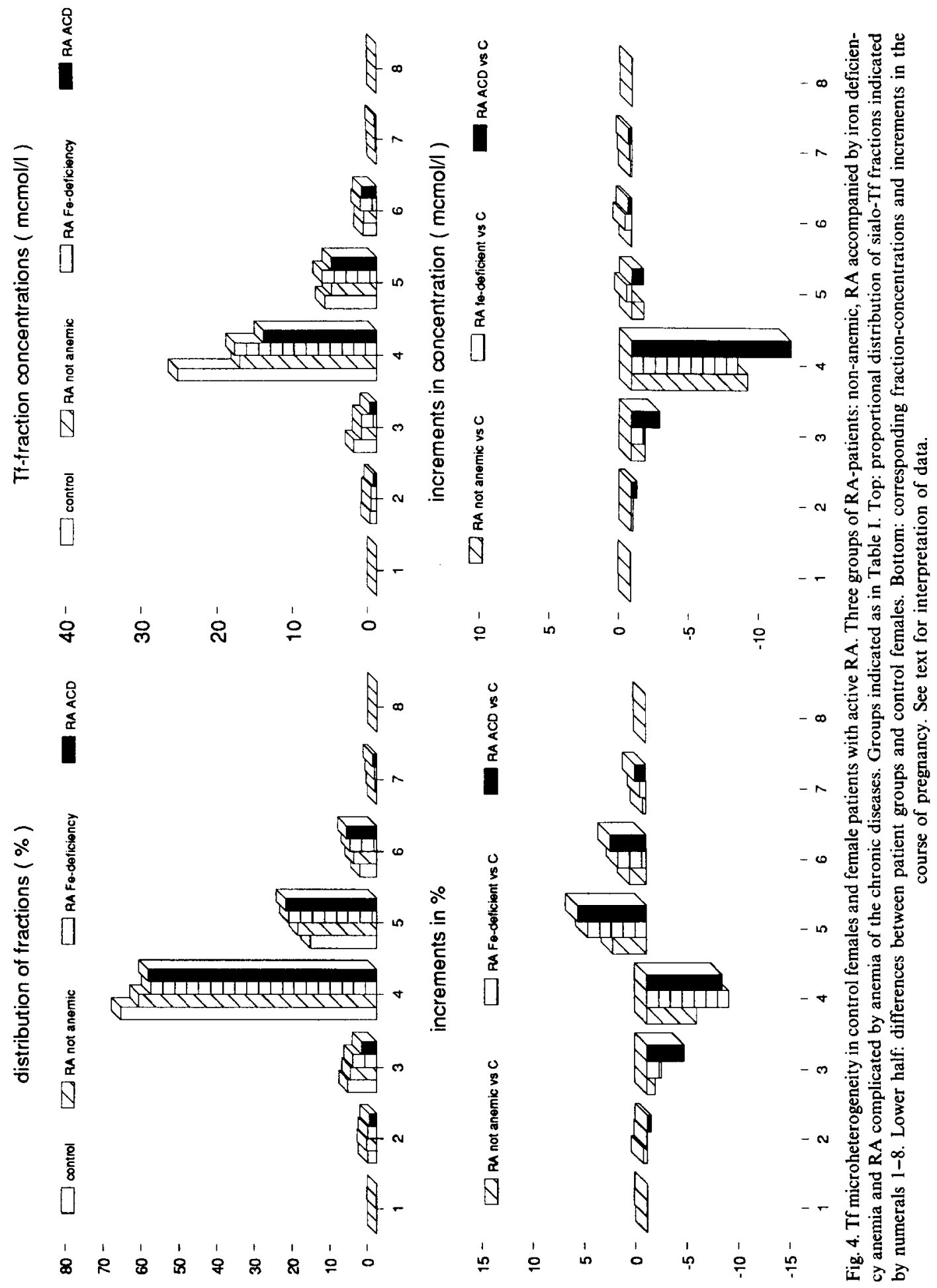


Also in all groups with established disease/physiological states the pattern of distribution was similarly remarkable in its uniformity. Variation in microheterogeneity can therefore not just be the result of random variation. The consistency of isoelectric focusing patterns characterizes the system in a broad sense, demonstrating that the process must be governed by a very potent control system, although the mechanism, apart from identification of constituting elements, is still beyond our perception.

A second conclusion within the same context refers to the coupling between protein and glycan synthesis. Results on sera from pregnant women already indicate that protein core synthesis and change of glycosylation towards higher branched glycans are not linked, since the increment in the forms with tri- and tetra-antennary chains correlated better with duration of gestation than with concentration of total Tf (Fig. 5). Women on oral contraceptives attain a lower level of change in microheterogeneity when compared to that stage in pregnancy where total Tf concentrations are similar, which indicates that changes in microheterogeneity and $\mathrm{Tf}$ protein synthesis are not linked. The most persuasive argument is provided in the results from the RA-group. In acute and chronic inflammatory conditions increased synthesis of acute phase proteins is invariably accompanied by changes in the microheterogeneity spectrum, at the least not incompatible with a linkage. Tf being a negative acute phase protein [24], it provides the model in which the effect of protein synthetic rate on $\mathrm{N}$-glycosylation is dissociated from the effect of the acute phase response on glycosylation. The microheterogeneity pattern can be changed markedly even when protein synthesis is significantly depressed. Therefore a fixed linkage between Tf protein synthetic rate and glycosylation pattern can be denied.

Hypothesis on the role and rationale of glycosylation changes viz-a-viz physiological and pathophysiological requirements for iron

Having concluded that observed changes in Tf microheterogeneity are not just related to random faults or a byproduct of increased synthetic rate, but the result of strictly controlled redirection of glycan synthesis, the next question that arises is whether these changes in Tf have any functional significance, or is Tf simply an innocent bystander as it happens to pass the synthetic machinery that has been adjusted for other reasons. To address this question results are reviewed in their pathophysiological contexts.

\section{Pregnancy}

The adaptation of the microheterogeneity pattern in the course of pregnancy in timing closely coincides with the major changes in iron metabolism as they occur during pregnancy. The increased demand of the fetoplacental unit and maternal bone marrow for iron is partly counterbalanced by cessation of menstruation. The remainder is partly met by increased iron absorption from the gut. The cumulated demand for iron may increase to above $8 \mathrm{mg} /$ day, which exceeds the uptake rate from the gut, therefore mobilization of iron from storage sites is an important mechanism in pregnancy, particularly in the later stages. The correlation between the 


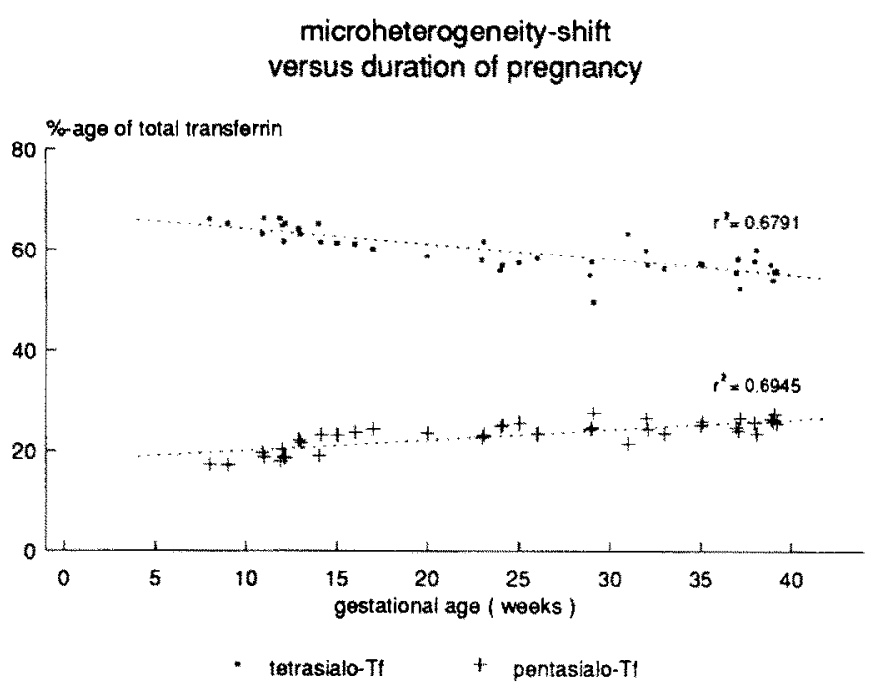

microheterogeneity-shift versus concentration total Tf

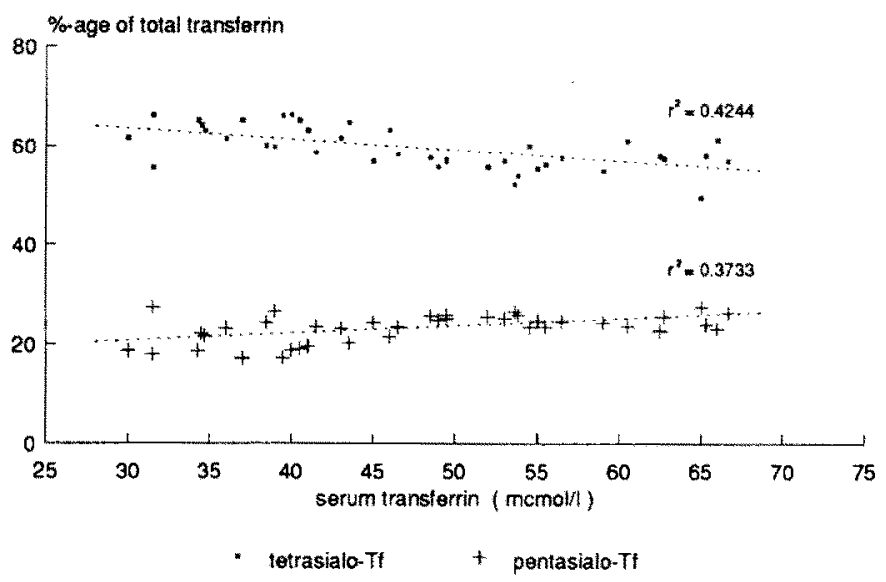

Fig. 5. Correlation diagrams displaying redirection of $\mathrm{Tf}$ glycan synthesis related to duration of pregnancy (top) and to total $\mathrm{Tf}$ concentration (bottom). Difference in correlation to duration significantly higher than correlation to Tf concentration; $P<0.001$.

increment in the production of highly sialylated $\mathrm{Tfs}$ and combined daily maternal and fetoplacental iron needs, combined with the absence of correlation with the production of tetrasialoTf, is striking (Fig. 6). As both major iron requiring processes, i.e. maternal erythroblast iron uptake and iron uptake on the maternal side of the placenta have been shown to be dependent on the interaction between $\mathrm{Tf}$ and the Tf receptor, the obvious conclusion is to relate these changes in microheterogeneity 
Microheterogeneity in pregnancy

in relation to iron requirement

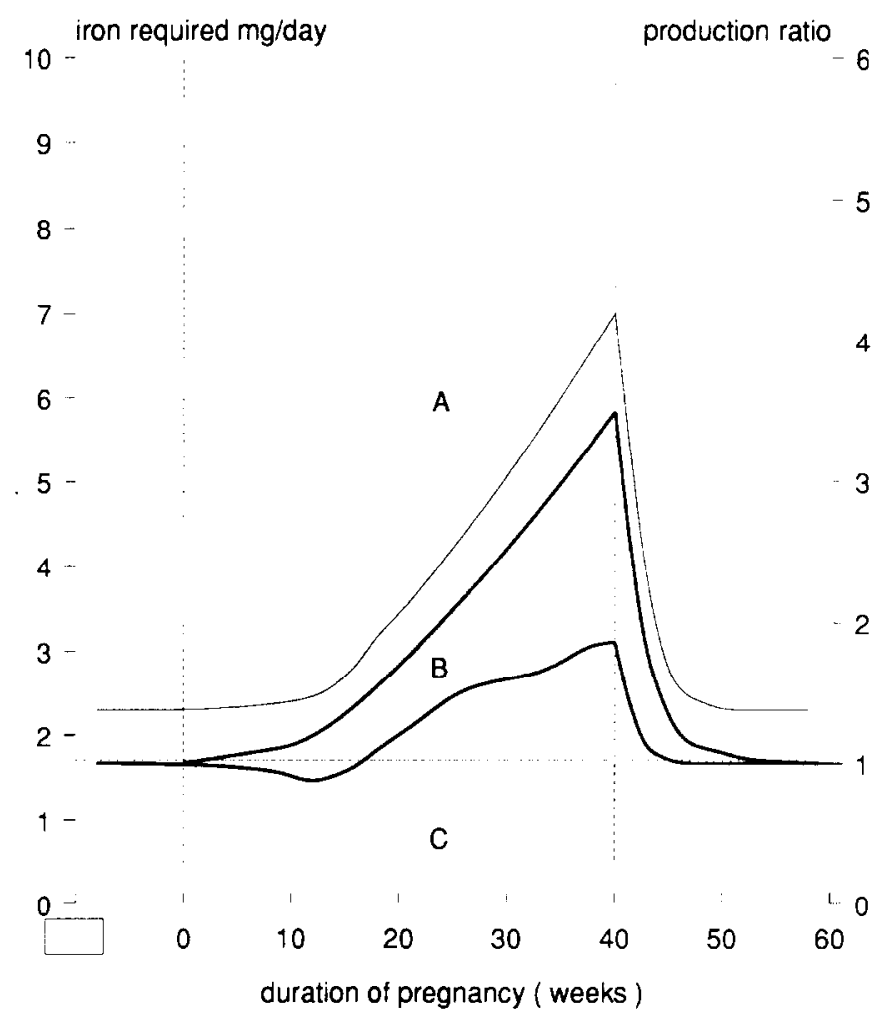

Fig. 6. Iron requirements during pregnancy and Tf microheterogeneity. (A) Iron requirement in $\mathrm{mg} /$ day. (B) pentasialo-Tf production ratio. (C) Tetrasialo-Tf production ratio. Iron requirement increasing almost exponentially until term mostly as a result of the steadily increasing transplacental iron transport and the increase of maternal red cell mass occurring predominantly in the third trimester. Shifts in microheterogeneity plotted as ratio between production before and at time of gestation and corrected for increased vascular fluid mass.

to increased iron fluxes. This concept of redirection of iron fluxes through modulation of functional characteristics of the molecule without affecting the protein moiety and independent from its rate of production is one that has a certain attraction, but admitted, coincidental concurrence can certainly not be excluded and the concept must await experimental verification. However, somc additional weight is added to the argument by observations in other diseases (see paragraphs on iron deficiency anemia and RA). Also in our department we have completed studies on apical microvillous surface membrane vesicles isolated by a modified Smith-procedure [25], the modification involving a choatropous treatment. These vesicles were incubated with tetra- and hexasialo-Tf isolated by preparative isoelectric focusing of genetically 
homogeneous Tf [5]. Scatchard analyses show that at least at low concentrations the hexasialo-Tf has a higher affinity for membrane vesicles prepared from term placenta villi than the tetrasialo-Tf (preliminary data, not shown).

\section{Iron deficiency anemia}

Iron deficiency is a condition that can be simply defined as a state of global physical demand for iron in the organism. Having postulated a functional role for the change in glycosylation in iron metabolism via modification of functional characteristics of $\mathrm{Tf}$ a dilemma is thus created: what rationale is there to account for the fact that changes in obviously the pre-eminently iron demanding condition are less prominent than in pregnancy?

The answer may lie in both the nature and the site of generation of the signal to adapt to the stress the organism is subjected to in each particular condition. In irondeficiency the matter is above all to absorb more iron from the gut and not so much to redistribute as to distribute any of the available iron as all tissues are devoid of iron. Tf plays no active role in absorption of iron from the intestines [3], denying a role for changes in Tf substructure in it as well. Secondly, the single most important site of production of Tf that ends up in the circulation is the liver, which itself through the Iron Responsive Elements incorporated in the genetic control of Tf protein synthesis turns the signal for synthesis on and off. On the contrary in pregnancy in essence the fetus, through regulation of the hormonal milieu in concert with the placenta, controls the whole range of maternal metabolic, hematologic and even cardiovascular adaptations to pregnancy [26]. Also, in the absence of anemia increased erythropoiesis is forced upon her, dictated by the fetoplacental unit. Even more so than in iron deficiency iron must be lured away from storage sites or preferentially be taken up by other organs than those involved in storage, explaining the logic for a physiological mechanism different to that in iron deficiency anemia.

\section{Rheumatoid arthritis (RA)}

Referring to the relative conservation of the production of highly sialylated Tfs, the above postulated facilitation of iron fluxes to the bone marrow through $\mathrm{Tf}$ glycan modulation can also be incorporated in the interpretation with respect to RA. In view of the high correlation of disease activity and shift in microheterogeneity pattern, the conservation of highly branched glycans may form part of a compensatory mechanism in anemias accompanying RA, attempting to facilitate a decreased erythroblast iron availability which has been postulated to be a major contributing factor in this condition. This decreased iron availability may rely on inhibitory effects of cytokines such as the interleukins and TNF, factors that have been shown to correlate highly with disease activity $[26,27]$. The same mechanism would of course also be operable in the disease stages not accompanied by ACD, but then be compensated for. In ACD this compensation appears to finally fall short. Of course this notion is based on correlations only and one could just as reasonably turn the argument around. For instance it can be postulated that anemia of the chronic diseases arises when the tetra-sialo-Tf concentration falls below a critical level. 


\section{Conclusion}

Clearly experimental data are needed. A convenient method for isolation of microheterogeneous forms of Tf has been developed [5] enabling us to study effects of different fractions on various cell types, but unfortunately a synthetic model is lacking at present. A cell culture model will have to be based on hepatocytes as the liver is the main source of $\mathrm{Tf}$ in the circulation. Unfortunately, the hepatocellular carcinoma cell lines PLC/PRF/5, HepG2 and Hep3B produce Tfs with unusual acidic isoelectric points (own observations, results not shown) and for that reason unsuitable for studying effects of hormonal changes in pregnancy, effects of mediators of the acute phase response or the effects of iron deficiency. For the moment we have relied on observations in physiological and pathological states and analyzed data with careful reference to the conditions they intrinsically define.

In conclusion, our results show that the glycosylation of $\mathrm{Tf}$ is a strictly controlled process, both in the physiological states and in disease. The microheterogeneity pattern is determined independently from $\mathrm{Tf}$ protein synthetic rate. Estrogens and possibly more so progestagens can influence microheterogencity. Mediators of the acute phase response can clearly be involved as well, experimental verification is however difficult due to the absence of an in vitro model.

In pregnancy an increase in $\mathrm{Tf}$ concentration is accompanied by redirection of glycan synthesis to the highly sialylated and highly branched glycans. The close correlation of this phenomenon throughout pregnancy with iron requirements as defined largely by transplacental iron transport and bone marrow iron consumption led us to postulate that this redefinition of the microheterogeneity pattern may function as a means to modulate internal iron fluxes between sites of storage and utilization. Similarly this proposition can be applied to RA, where a similar shift in the pattern is observed, in which case the mechanism may serve as a means of compensation for the decreased erythroblast iron availability which characterizes this condition. Experimental work probing these conclusions is in progress.

\section{Acknowledgements}

The authors wish to thank J.H.C. Wallenburg, A. Helbing, G. Vreugdenhil, A.J.G. Swaak and F.M.N. Zuijderhoudt for kindly providing blood samples and basic patient details and B.G. Blijenberg for ferritin and Tf-assays.

\section{References}

1 Aisen P, Listowsky I. Iron transport and storage proteins. Annu Rev Biochem 1980;49:357-393.

2 Huebers H, Finch CA. The physiology of transferrin and tranferrin receptors. Physiol Rev 1987;67:520-582.

3 de Jong G, van Dijk JP, van Eijk HG. The biology of transferrin. Clin Chim Acta 1990;190:1-46.

4 de Jong G, van Eijk HG. The microheterogeneity of human serum transferrin. A biological phenomenon studied by isoelectric focusing in immobilized $\mathrm{pH}$ gradients. Electrophoresis 1988;9(2):589-598.

5 de Jong G, van Noort WL, van Eijk HG. Carbohydrate analysis of transferrin subfractions isolated by preparative isoelectric focusing in immobilized pH gradients. Electrophoresis 1992;13:225 228. 
6 Jaeken J, van Eijk HG, van der Heul C, Corbeel L, Eeckels R, Eggermont E. Sialic acid-deficient serum- and cerebrospinal fluid transferrin in a newly recognized genetic syndrome. Clin Chim Acta 1984;144:245-246.

7 Stibler H, Jaeken J. Carbohydrate deficient serum transferrin in a new systemic hereditary syndrome. Arch Dis Child 1990;65:107-111.

8 Bleasby AJ, Knowles JC, Cooke $\mathrm{NJ}_{*}$ Microheterogeneity of $\alpha_{1^{-a c i d}}$ glycoprotein: lack of discrimination between benign and malignant inflammatory disease of the lung. Clin Chim Acta 1985;150:231-235.

9 Rosendah K, Waldenlind L, Onica D. Microheterogeneity of serum alkaline phosphatase isoenzymes as revealed by isoelectric focusing. Clin Chim Acta 1987;168:297-306.

10 Biou D, Chanton P, Konan D et al. Microheterogeneity of the carbohydrate moiety of human alpha1-acid glycoprotein in two benign liver diseases: alcoholic cirrhosis and acute hepatitis. Clin Chim Acta 1989;186:59-66.

11 Arnett FC, Edworthy SM, Bloch DA et al. The American Rheumatism Association 1987 Revised Criteria for the classification of Rheumatoid Arthritis. Arth Rheum 1988;31:315-324.

12 Cartwright GE, Lee GR. The anemia of chronic disorders. Br J Haematol 1971;21:147-152.

13 Vreugdenhil G, Baltus CAM, van Eijk HG, Swaak AJG. Anemia of Chronic Disease: diagnostic significance of erythrocyte and serological parameters in iron deficient rheumatoid arthritis patients. Br J Rheumatol 1990;29:105-110.

14 Dykes DD, Polesky HF. Review of isoelectric focusing for Gc, PGM, Tf and Pi subtypes: population distributions. CRC Crit Rev Clin Lab Sci 1984;20:115-151.

15 Ritchie RF. Automated Immunoprecipitation Analysis of Serum Proteins. In: Putnam F, ed. The Plasma Proteins (2nd edn) 1975:2:375.

16 Raynes J. Variation in the relative proportion of microheterogeneous forms of plasma glycoprotein in pregnancy and disease. Biomedicine 1982;36:77-86.

17 Birgegard G, Hällgren $R$, Caro J. Serum erythropoietin in rheumatoid arthritis and other inflammatory arthritides: relationship to anemia and the effect of anti-inflammatory treatment. Br $\mathrm{J}$ Haematol 1987;65:479-483.

18 Mackiewicz A, Pawlowski T, Mackiewicz-Pawlowska A, Wiktorowicz K, Mackiewicz S. Microheterogeneity forms of alpha-1 acid glycoprotein as indicators of rheumatoid arthritis activity. Clin Chim Acta 1987;163:185-190.

19 de Jong G, van Eijk HG. Functional properties of the carbohydrate moiety of human transferrin. Int J Biochem 1989;21(3):253-263.

20 Berger EG, Buddecke E, Kamerlingh JP, Kobata A, Paulsson JC, Vliegenthart JFG. Structure, biosynthesis and functions of glycoprotein glycans. Experentia 1982;38:1129-1258.

21 Kornfeld R, Kornfeld S. Assembly of asparagine-linked oligosaccharides. Annu Rev Biochem $1985 ; 54: 631-664$.

22 Roth J. Subcellular organization of glycosylation in mammalian cells. Biochim Biophys Acta 1987;906:405-436.

23 Schachter $\mathbf{H}$. Glycoproteins: their structure, biosynthesis and possible clinical implications. Clin Biochem 1984;17:3-14

24 Kushner 1. The phenomenon of the acute phase response. Ann NY Acad Sci 1982;389:39-48.

25 Smith CH, Nelson MD, King BF, Donohue TM, Ruzycki SM, Kelly LK. Characterization of a microvillous membrane preparation from human placental syncytiotrophoblast: a morphologic, biochemical and physiologic study. Am J Obstet Gynecol 1977;128:190-196.

26 Scott DE. anemia in pregnancy, In: Wynn RM, ed. Obstetrics and gynaecology annual. New York: Appleton-Century-Crofts $1972 ; 1 ; 219-244$.

27 Malkowsky M, Sondel PM, Strober W, Dalgleish AG. The interleukins in acquired disease. Clin Exp Inmunol 1988;74:1151-161.

28 Swaak AJG, van Rooijen A, Niewenhuis E, Aarden LA. Interleukin-6 in synovial fluid and serum of patients with rheumatic diseases. Scand J Rheumatol 1988;17:469-474. 